\title{
Increased investment in gametocytes in asymptomatic Plasmodium falciparum infections in the wet season
}

Colins O. Oduma ${ }^{1,2}$, Sidney Ogolla2, Harrysone Atieli ${ }^{3,4}$, Bartholomew N. Ondigo ${ }^{1,5}$, Ming-Chieh Lee ${ }^{6}$, Andrew K. Githeko ${ }^{4}$, Arlene E. Dent ${ }^{7}$, James W. Kazura ${ }^{7}$, Guiyun Yan $^{6}$ and Cristian Koepfli ${ }^{8^{*}}$ (D

\begin{abstract}
Background: Transmission stemming from asymptomatic infections is increasingly being recognized as a threat to malaria elimination. In many regions, malaria transmission is seasonal. It is not well understood whether Plasmodium falciparum modulates its investment in transmission to coincide with seasonal vector abundance.

Methods: We sampled 1116 asymptomatic individuals in the wet season, when vectors are abundant, and 1743 in the dry season, in two sites in western Kenya, representing different transmission intensities (Chulaimbo, moderate transmission, and Homa Bay, low transmission). Blood samples were screened for $P$. falciparum by qPCR, and gametocytes by pfs25 RT-qPCR.

Results: Parasite prevalence by qPCR was 27.1\% (Chulaimbo, dry), 48.2\% (Chulaimbo, wet), 9.4\% (Homabay, dry), and $7.8 \%$ (Homabay, wet). Mean parasite densities did not differ between seasons ( $P=0.562)$. pfs 25 transcripts were detected in 119/456 (26.1\%) of infections. In the wet season, fewer infections harbored detectable gametocytes (22.3\% vs. 33.8\%, $P=0.009$ ), but densities were 3 -fold higher (wet: 3.46 transcripts/uL, dry: 1.05 transcripts/uL, $P<$ 0.001). In the dry season, $4.0 \%$ of infections carried gametocytes at moderate-to-high densities likely infective (> 1 gametocyte per 2 uL blood), compared to $7.9 \%$ in the wet season. Children aged 5-15 years harbored $76.7 \%$ of infections with gametocytes at moderate-to-high densities.

Conclusions: Parasites increase their investment in transmission in the wet season, reflected by higher gametocyte densities. Despite increased gametocyte densities, parasite density remained similar across seasons and were often below the limit of detection of microscopy or rapid diagnostic test, thus a large proportion of infective infections would escape population screening in the wet season. Seasonal changes of gametocytemia in asymptomatic infections need to be considered when designing malaria control measures.
\end{abstract}

Keywords: Plasmodium falciparum, Transmission, Gametocyte, Asymptomatic, Season

\footnotetext{
* Correspondence: ckoepfli@nd.edu

${ }^{8}$ Eck Institute for Global Health and Department of Biological Sciences,

University of Notre Dame, Notre Dame, IN 46556-0369, USA

Full list of author information is available at the end of the article
}

(c) The Author(s). 2021 Open Access This article is licensed under a Creative Commons Attribution 4.0 International License, which permits use, sharing, adaptation, distribution and reproduction in any medium or format, as long as you give appropriate credit to the original author(s) and the source, provide a link to the Creative Commons licence, and indicate if changes were made. The images or other third party material in this article are included in the article's Creative Commons licence, unless indicated otherwise in a credit line to the material. If material is not included in the article's Creative Commons licence and your intended use is not permitted by statutory regulation or exceeds the permitted use, you will need to obtain permission directly from the copyright holder. To view a copy of this licence, visit http://creativecommons.org/licenses/by/4.0/. The Creative Commons Public Domain Dedication waiver (http://creativecommons.org/publicdomain/zero/1.0/) applies to the data made available in this article, unless otherwise stated in a credit line to the data. 


\section{Background}

Malaria transmission stemming from asymptomatic individuals has gained attention as an increasing number of countries aims for malaria elimination rather than control. Most tools for intervention, such as bed nets, or indoor residual spraying (IRS), were developed and tested to reduce the number of clinical cases [1]. Their impact on asymptomatic infections and their transmission potential is little understood. Approaches to specifically identify and treat asymptomatic infections, such as reactive case detection [2], mass drug administration [3], or combinations thereof [4] are increasingly trialed or implemented. A better understanding of how to best use them to minimize transmission from asymptomatic carriers is needed.

In many settings with pronounced seasonality in rainfall, Anopheles mosquitoes are sparse in the dry season as opposed to wet season where they are plentiful, resulting in transmission primarily occurring during and shortly after the wet season [5-9]. It is not known how far $P$. falciparum adapts its transmission potential to changes in vector abundance across seasons. Adaptions to increase transmission potential when chances for onward transmission are high could maximize the fitness of the parasite population. Understanding such adaptations are crucial when introducing transmissionreducing interventions.

Over the course of the red blood cell cycle, a small proportion of $P$. falciparum parasites develop into gametocytes, the sexual form of the parasite [10]. A mosquito blood meal needs to contain at least one female and one male gametocyte to be infective [11, 12]. The ingested gametocytes develop into oocysts and after approximately two weeks, into sporozoites that are transmitted to the next vertebrate host [13]. P. falciparum gametocytes exist in five morphologically distinguishable stages [14]. Early ring stage gametocytes circulate in peripheral blood [15] while late stages I-IV sequester for 7 to 12 days in inner organs including bone marrow and spleen until maturity $[12,16,17]$. The mature stage $\mathrm{V}$ gametocytes re-enter the peripheral circulation where they require an additional 3 days to become fully infective [18, 19]. Stage V gametocytes remain in the circulation for a mean period of 6.4 days to a maximum of 3 weeks [17]. Due to the sequestration of developing gametocytes, they are rarely detected in peripheral blood during the first two weeks following sporozoite inoculation.

A large proportion of all $P$. falciparum infections remain asymptomatic. Untreated infections can persist for several months [20-22]. During this time, parasite densities fluctuate and are often below the limit of detection by microscopy. Transmission stemming from asymptomatic infections is a key obstacle for malaria control and elimination. Such subpatent $P$. falciparum gametocyte carriers have the potential to infect mosquitoes [23-25], though their contribution to transmission in different settings is not known [26]. A previous study in western Kenya found asymptomatic individuals to be more infective than clinical cases [27]. Even after antimalarial treatment, gametocytes may continue to circulate for up to 2-3 weeks [28]. Gametocyte densities are an important measure to predict the infectiousness of humans to mosquitoes [23-25], and thus useful for evaluating the effects of interventions that aim to reduce transmission [26].

Gametocyte density in the blood is governed by the conversion rate, i.e., the proportion of early ring stage parasites committed to sexual vs. asexual development. Changes in the density of mature gametocyte could be achieved through different strategies, e.g. a change of the conversion rate, growing a higher density of asexual parasites before any gametocytes develop, longer circulation of mature gametocytes, or a combination of these factors [29]. In all cases, a higher density of gametocytes will increase transmission if vectors are present. On the other hand, the investment in gametocytes is lost if gametocytes are not taken up by mosquitoes. The factors affecting the conversion rate are not well understood. In laboratory culture and rodent malaria models, factors such as high parasite density [30] and drug pressure [31] have been found to impact gametocyte conversion. Few studies have measured the conversion rate directly in natural infections and observed pronounced variation [32-34].

Areas of western Kenya experience perennial malaria transmission with peaks in vector density and transmission coinciding with seasonal rains in April-August and October-November [5, 35]. It is not known whether asymptomatic $P$. falciparum infections modulate the investment in gametocytes to coincide with the appearance of vectors at the start of transmission period.

To understand seasonal changes in gametocyte carriage in sites of differential transmission intensity, we compared $P$. falciparum gametocyte densities in asymptomatic individuals between the dry and wet seasons in a low-transmission setting (Homa Bay) and a moderatetransmission setting (Chulaimbo) in western Kenya. Blood stage parasites were diagnosed by varATS qPCR, and mature female gametocytes were quantified using pfs 25 reverse transcriptase qPCR.

\section{Methods}

\section{Study sites and participants}

2859 asymptomatic individuals were sampled in crosssectional surveys in the dry season $(n=1116)$ between January and March 2019, and wet season $(n=1743)$ between June and August 2019 in Western Kenya, in Homa Bay (low transmission) and Chulaimbo (moderate 
Table 1 Demographic and parasitological characteristics of study participants

\begin{tabular}{|c|c|c|c|c|c|c|}
\hline & \multicolumn{2}{|l|}{ Chulaimbo } & & \multicolumn{2}{|l|}{ Homa Bay } & \\
\hline & $\begin{array}{l}\text { dry } \\
N=262\end{array}$ & $\begin{array}{l}\text { wet } \\
N=419\end{array}$ & & $\begin{array}{l}\text { dry } \\
N=854\end{array}$ & wet $N=1324$ & \\
\hline \multicolumn{7}{|l|}{ Demographic data } \\
\hline Age group in years & $\mathrm{N}(\%)$ & N (\%) & & N (\%) & N (\%) & \\
\hline$<5$ & $45(17.2)$ & $62(14.8)$ & & $158(18.5)$ & $227(17.1)$ & \\
\hline $5-15$ & $75(28.6)$ & $173(41.3)$ & & $154(18.0)$ & $419(31.6)$ & \\
\hline$>15$ & $142(54.2)$ & $184(43.9)$ & & $542(63.5)$ & $678(51.2)$ & \\
\hline Female (\%) & $155(59.2)$ & $234(55.8)$ & & $578(67.7)$ & $840(63.4)$ & \\
\hline Parasitological data & & & $P$ value & & & $P$ value \\
\hline Parasite prevalence & $71 / 262(27.1 \%)$ & $202 / 419(48.2 \%)$ & $<0.0001^{*}$ & $80 / 854(9.4 \%)$ & 103/1324 (7.8\%) & 0.1921 \\
\hline Geometric mean parasite density & $7.79[3.07-19.7]$ & $11.7[6.80-20.2]$ & 0.8433 & $6.87[3.53-13.4]$ & $5.31[2.88-9.81]$ & 0.9638 \\
\hline Proportion subpatent infections ( $<100$ parasites $/ \mathrm{hL}$ ) & $48 / 71(67.6 \%)$ & 135/202 (66.8\%) & 0.9050 & $63 / 80(78.8 \%)$ & 83/103 (80.6\%) & 0.7595 \\
\hline Population gametocyte prevalence & $27 / 262(10.3 \%)$ & $50 / 419(11.9 \%)$ & 0.5140 & $24 / 854(2.8 \%)$ & 18/1324 (1.4\%) & $0.0162^{*}$ \\
\hline Proportion gametocyte positive infections & $27 / 71(38.0 \%)$ & $50 / 202(24.8 \%)$ & $0.0325^{*}$ & $24 / 80(30.0 \%)$ & 18/103 (17.5\%) & $0.0457^{*}$ \\
\hline Geometric mean pfs 25 density & $1.37[0.79-2.36]$ & $4.74[2.36-9.55]$ & $0.0181^{*}$ & $0.77[0.54-1.10]$ & $1.42[0.76-2.65]$ & 0.0638 \\
\hline
\end{tabular}

Numbers in brackets are $95 \%$ confidence intervals; asterisk $(*)$ indicate significant at $P<0.05$; densities are determined using T-test; and differences in prevalence using the Chi-Square test

transmission) (Table 1, Fig. 1). In these areas, P. falciparum is the primary malaria parasite species [36]. The study population included asymptomatic individuals aged 2 months to 99 years with no clinical symptoms. None of the study participants had been treated with antimalarial drugs within the three days prior blood sampling.

In Chulaimbo, Anopheles arabiensis is the primary vector. It is abundant in the wet season. An. gambiae s.s is the second predominant mosquito vector [5]. In Homa Bay, An. funestus has re-emerged as the predominant species following development of pyrethroid resistance [37]. According to the Kenya "End of Spray" Report (2018) [38], indoor residual spraying (IRS) in Homa Bay has resulted in reduction in malaria vector densities and sporozoite rates compared with Chulaimbo where IRS has not been implemented.

\section{Sample collection and processing}

$350-400 \mu \mathrm{L}$ of capillary blood was collected into EDTA microtainer tubes (Becton Dickinson, New Jersey, United States) by finger prick. For RNA preservation, $100 \mu \mathrm{L}$ of whole blood was transferred to a tube containing $500 \mu \mathrm{L}$ of RNAlater (Sigma-Aldrich, Missouri, United States) within $2 \mathrm{~h}$ of collection and stored at $-80^{\circ} \mathrm{C}$ until RNA extraction [39, 40]. The remaining blood was centrifuged, plasma removed and stored at $-20^{\circ} \mathrm{C}$. The red cell pellet was stored at $-20^{\circ} \mathrm{C}$ until DNA extraction.

\section{Molecular parasite screening and quantification}

DNA was extracted from $100 \mu \mathrm{L}$ blood using the Genomic DNA Extraction kit (Macherey-Nagel, Düren, Germany) and eluted in an equivalent volume of elution buffer. DNA was screened for P. falciparum using ultrasensitive qPCR that amplifies a conserved region of the var gene acidic terminal sequence (varATS) according to a previously published protocol [41]. The varATS gene assay amplifies $\sim 20$ copies/genome [41]. The qPCR results were converted to varATS copies/ $\mu \mathrm{L}$ using external standard curve of ten-fold serial dilutions (5-steps) of 3D7 P. falciparum parasites quantified by droplet digital PCR (ddPCR) [42]. The ddPCR thermocycling conditions, sequences and concentration of primers and probe are given in additional file 1. Asexual parasite densities were calculated by dividing varATS copy numbers by 20 , reflecting the approximate number of copies per genome.

For all the gametocytes assays, RNA was extracted using the pathogen Nucleic Acid Extraction kit (Macherey-Nagel, Düren, Germany) and eluted in $50 \mu \mathrm{L}$ elution buffer, i.e., RNA was concentrated two-fold during extraction. RNA samples were DNase treated (Macherey-Nagel, Düren, Germany) to remove genomic DNA that could result in a false positive pfs 25 signal [43]. A subset of RNA samples was tested by varATS qPCR, and all tested negative.

\section{Molecular gametocyte screening and quantification}

For gametocyte detection by quantitative reverse - transcription PCR (RT-qPCR), RNA was extracted from all $P$. falciparum qPCR-positive samples. Gametocytes were quantified by targeting female $p f s 25 \mathrm{mRNA}$ transcripts using one-step RT-qPCR assays (Alkali Scientific, Florida, United States). All qPCR conditions, sequences and concentration of primers and probes are given in additional file 1 . The $p f s 25 \mathrm{RT}-\mathrm{qPCR}$ results were converted 


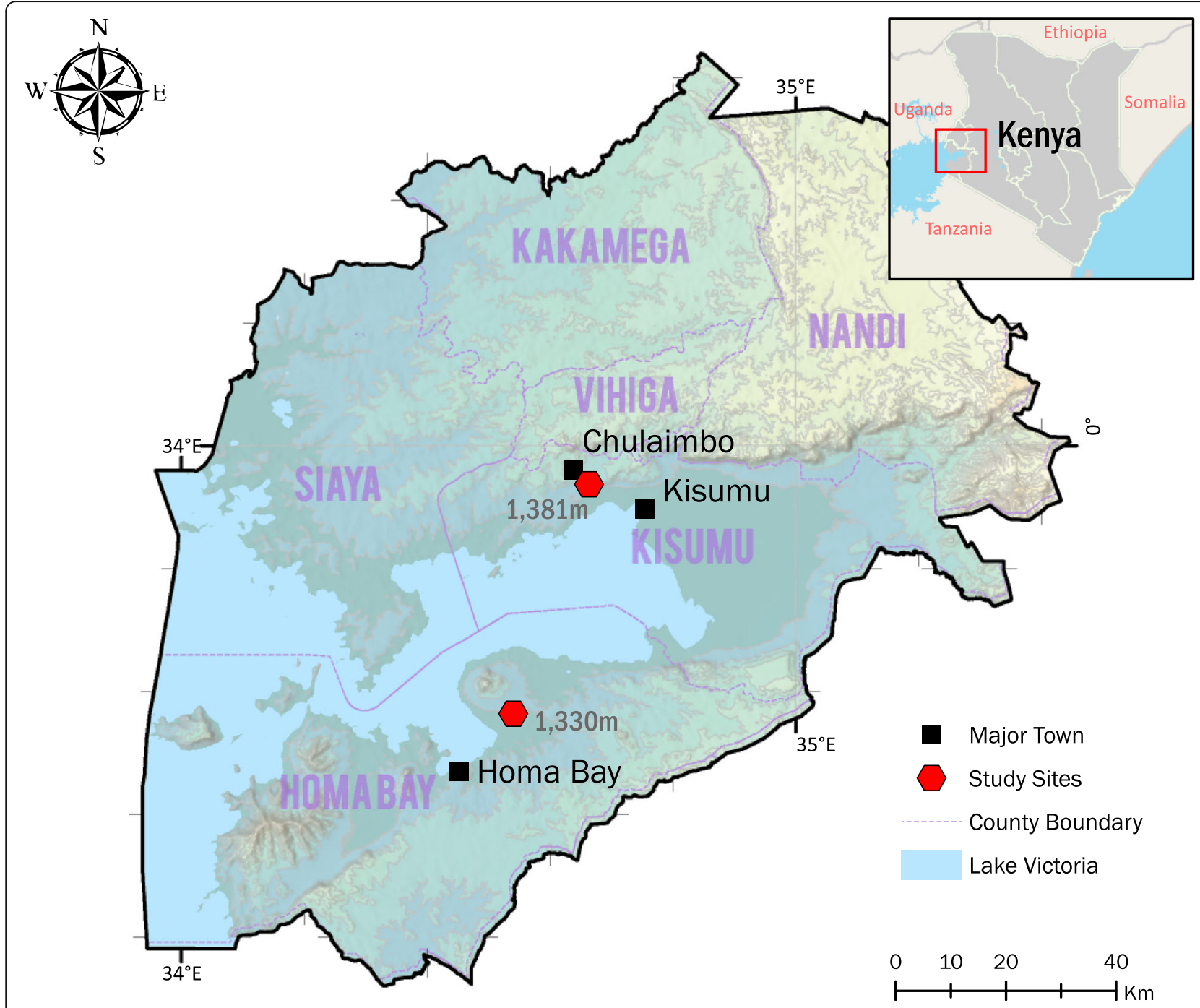

Fig. 1 Map of study sites

to $p f s 25$ transcript copies $/ \mu \mathrm{L}$ using external standard curve of ten-fold serial dilutions (5-steps) of DNA of 3D7 culture parasites quantified by ddPCR (additional file 1). Previous studies have shown that 10-20 pfs 25 transcripts are detected per gametocyte (reported as 90 transcripts/gametocyte when measured against a circular plasmid, which reflects a 5 to 10 -fold overestimate) $[40,42]$.

\section{Statistical analysis}

Parasite and gametocyte densities were $\log _{10}$ transformed and geometric means per $\mu \mathrm{L}$ blood calculated whenever densities were reported. The Shapiro-Wilk test and graphical normality was employed to determine normal distribution of data following log transformation. Differences in prevalence between seasons and sites were determined using the $x^{2}$ test. Differences in densities between seasons and sites were determined using T-test.
Differences in densities between age-groups were determined by ANOVA's Tukey's multiple comparisons test. Multivariable analysis was employed to determine association of age, site and season with asexual parasite and gametocyte positivity and density. The associations were investigated by linear and logistic regression analysis. Pearson's correlation test was conducted to establish the relationship between asexual parasite and gametocyte densities. Analysis was done in GraphPad Prism version 8 and STATA version 14 .

\section{Results}

Prevalence and density of $P$. falciparum infections

2859 samples with age distribution representative of the population were analyzed in this study. The demographic characteristics of the study participants are summarized in Table 1. 
Table 2 Parasitological characteristics of study participants by season

\begin{tabular}{|c|c|c|c|}
\hline & $\begin{array}{l}\text { dry } \\
N=1116\end{array}$ & $\begin{array}{l}\text { wet } \\
N=1743\end{array}$ & \\
\hline Parasitological data & & & $P$ value \\
\hline Parasite prevalence & $151 / 1116(13.5 \%)$ & $305 / 1743(17.5 \%)$ & $0.0047^{*}$ \\
\hline Geometric mean parasite density & $7.29[4.18-12.7]$ & $8.98[5.92-13.6]$ & 0.5620 \\
\hline Proportion subpatent infections ( $<100$ parasites $/ \mu \mathrm{L}$ ) & $111 / 151(73.5 \%)$ & $218 / 305(71.5 \%)$ & 0.6483 \\
\hline Population gametocyte prevalence & $51 / 1116(4.6 \%)$ & $68 / 1743(3.9 \%)$ & 0.3826 \\
\hline Proportion gametocyte positive infections & $51 / 151(33.8 \%)$ & $68 / 305(22.3 \%)$ & $0.0086^{*}$ \\
\hline Geometric mean pfs 25 density & $1.05[0.76-1.46]$ & $3.46[2.00-5.97]$ & $<0.0001^{*}$ \\
\hline
\end{tabular}

Across both sites, parasite prevalence by qPCR was $13.5 \%$ in the dry season and $17.5 \%$ in the wet season (Table 2, site specific-data presented in Table 1). In both seasons, prevalence of $P$. falciparum infection was significantly higher in Chulaimbo than Homa Bay (wet: $48.2 \%$ vs. $7.8 \%, P<0.001$, dry: $27.1 \%$ vs. $9.4 \%, P<0.001$, Table 1). In Chulaimbo, the prevalence was significantly higher in the wet season $(P<0.001$, Table 1$)$, but it did not differ between seasons in Homa Bay $(P=0.192$, Table 1). Across all surveys, prevalence was higher in males than females $(21.4 \%$ vs. $12.8 \%, P<0.001)$. Schoolage children ( $5-15$ years) were at highest risk of infection (Fig. 2).

Parasite densities by qPCR did not differ between seasons (Table 1, Table 2). Across all surveys, parasite densities differed significantly between age groups $(P<0.001$, Fig. 2). The densities peaked in children aged 5-15 years with a mean of 20.8 parasites $/ \mu \mathrm{L}$ ( $95 \%$ confidence interval [CI95]: 12.6-34.5), and thus were 6-fold higher than in adults aged $>15$ years (3.3 parasites/ $\mu \mathrm{L}, \mathrm{CI} 95: 2.1-5.1$ ).

\section{Proportion of gametocyte positive infections and gametocyte density}

Across all surveys, gametocytes were detected in 119/ 2859 (4.2\%) individuals. The population gametocyte prevalence differed significantly between sites across seasons (Table 1, wet: $P<0.001$, dry: $P<0.001$ ), and ranged from $1.4 \%$ in Homa Bay in the wet season to $11.4 \%$ in Chulaimbo in the wet season $(P<0.001$, Table 1$)$. The proportion of all individuals with blood stage parasites who were positive for gametocytes (the proportion of gametocyte positive infections) was significantly higher in the dry season $(33.8 \%)$ than in wet season $(22.3 \%, P=$ 0.009 , Table 2 , site-specific data presented in Table 1), but no difference was observed between sites (wet: $P=$ 0.149 , dry: $P=0.298$ ).

The proportion of parasite and gametocyte carriers, and proportion of gametocyte positive infections was highest in school-age children aged 5-15 years across seasons and sites (Fig. 2). pfs 25 transcripts $/ \mu \mathrm{L}$ differed significantly between age groups $(P=0.004$, Fig. 2$)$. The

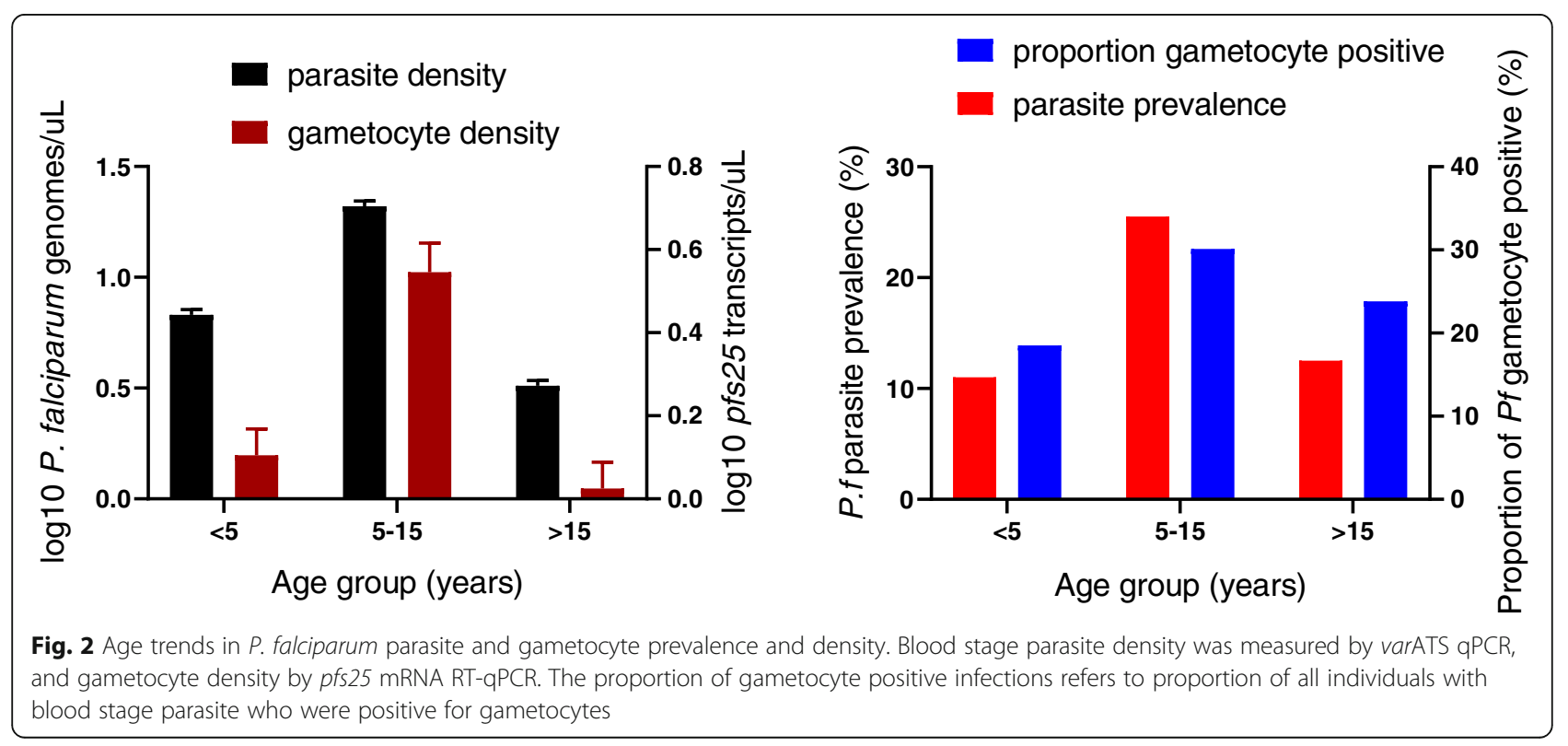




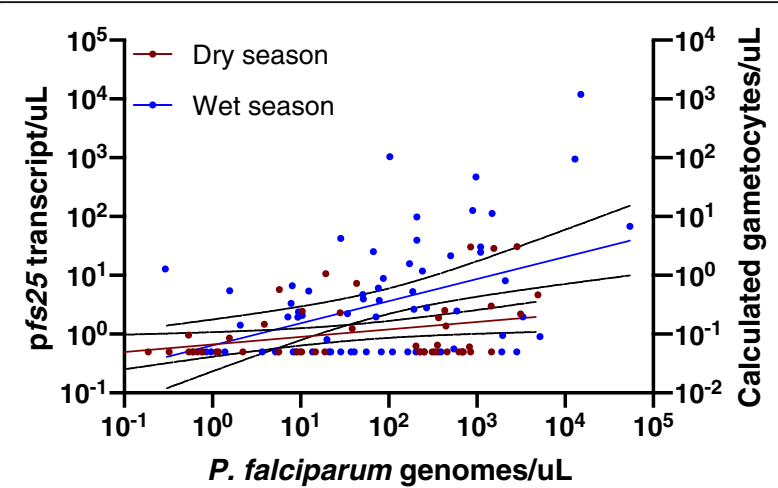

Fig. 3 Correlation between $P$. falciparum parasite and gametocyte densities across seasons. Black lines show 95\% confidence intervals. The left $Y$-axis shows the number of pfs 25 transcripts measured by RT-qPCR. The right $Y$-axis shows the calculated number of gametocytes, assuming 10 transcripts per gametocyte $[40,42]$

transcript copies $/ \mu \mathrm{L}$ peaked in children aged $5-15$ years with a mean of 3.6 transcripts $/ \mu \mathrm{L}$ (CI95: 2.0-6.4), and thus were 3 -fold higher than in adults aged $>15$ years (1.1 transcripts/ $\mu \mathrm{L}$, C195: 0.8-1.5). The correlation between varATS copy numbers and $p f s 25$ transcripts was moderate, but highly significant $(\mathrm{R}=0.36, P<0.001$, Fig. 3). Likewise, the probability to detect gametocytes was correlated with parasite density. Each 10-fold increase in genome copies resulted in 3.23-fold higher odds in carrying $p f s 25$ transcripts.

\section{Seasonal differences in gametocyte carriage}

Seasonal patterns in gametocyte carriage were similar in Homa Bay and Chulaimbo. Thus, results are presented for both sites combined (Table 2, site-specific data in Table 1). The proportion of gametocyte positive infections was significantly higher in the dry season $(33.8 \%$, $51 / 151)$ compared to the wet season $(22.3 \%, 68 / 305, P=$ 0.009 , Table 2). In contrast, mean gametocyte densities were 3-fold higher in the wet season (wet: 3.46 pfs 25 transcripts/ $\mu \mathrm{L}$ (CI95: 2.0-6.0), dry: 1.05 pfs 25 transcripts/ $\mu$ L (CI95: 0.8-1.5), $P<0.001$, Fig. 3, Fig. 4), even though parasite densities did not differ across seasons (wet: 8.98 varATS copies/genome (CI95: 5.9-13.6), dry: 7.29 varATS copies/genome (CI95: 4.2-12.7), $P=0.562$, Table 2). The difference in $p f s 25$ transcript numbers between seasons remained highly significant when including log-transformed parasite densities as a predictor in multivariable analysis (Table 3). No interaction was observed between parasite density and the probability that an individual carried gametocytes, and season $(P=$ 0.739).

Pronounced variation in gametocyte carriage was observed among infections, with many medium- or highdensity infections not carrying any detectable

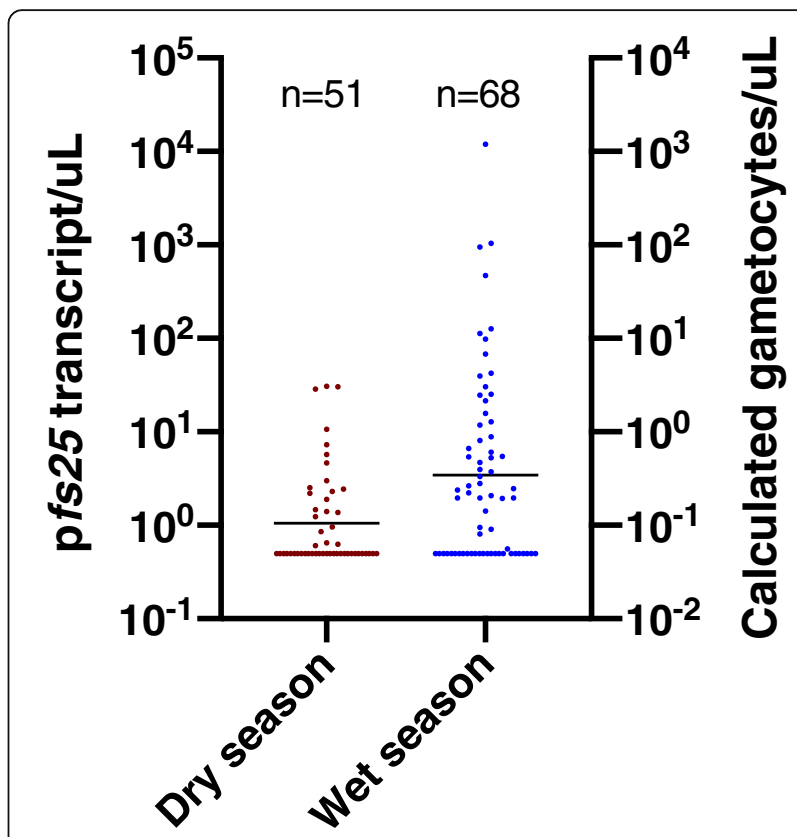

Fig. 4 P. falciparum gametocyte densities across seasons. The left $Y$-axis shows the number of pfs 25 transcripts measured by RTqPCR. The right $Y$-axis shows the calculated number of gametocytes, assuming 10 transcripts per gametocyte $[40,42]$. The black line shows geometric mean pfs 25 densities

gametocytes. Among infections with a density of $>2000$ varATS copies $/ \mu \mathrm{L}$ (corresponding to $>100$ parasites $/ \mu \mathrm{L}$ ), in the wet season $60.0 \%(24 / 40)$ carried gametocytes versus $36.8 \%(32 / 87)$ in the dry season $(P=0.014)$. Very few individuals carried gametocytes at high densities. For example, across both surveys, only 30 individuals carried $p f s 25$ transcripts at densities $>5$ transcripts/ $\mu \mathrm{L}$ (corresponding to approximately 1 gametocyte per $2 \mu \mathrm{L}$ of blood), 6 in the dry and 24 in the wet season. Among them, $4 / 6$ and 19/24 were school-age children aged 515 years.

In multivariable analysis, only parasite density and season were found to be significantly associated with the probability that an individual was gametocyte positive (Table 3). Age group $(P=0.195)$, sex $(P=0.214)$, and site $(P=0.364)$ were not associated. Likewise, gametocyte

Table 3 Multivariable predictors of gametocyte positivity and density

\begin{tabular}{lll}
\hline pfs25 positivity & aOR & P value \\
\hline log10 Pf copies & 0.53 & $<0.001$ \\
Wet season & -0.716 & 0.002 \\
log10 pfs25 density & Coefficient & $\boldsymbol{P}$ value \\
log10 Pf copies & 0.246 & $<0.001$ \\
Wet season & 0.42 & 0.004 \\
\hline
\end{tabular}

Only parasite density (log10 transformed) and season were found to be significant predictors for the probability that an infection was positive for pfs 25 , and for pfs 25 density. aOR = adjusted odds ratio 
density was only significantly associated with parasite density and season, but not site $(P=0.063)$, age group $(P=0.733)$, or sex $(P=0.611)$ (Table 3$)$.

\section{Gametocyte carriage among patent and subpatent infections}

A sensitivity of 100 blood stage parasites $/ \mu$ L (i.e. asexual parasites and gametocytes) was assumed to determine the proportion of infections that could be detected by Rapid Diagnostic Test (RDT) or light microscopy. Given this threshold, $72.1 \%(329 / 456)$ of all infections were subpatent across all surveys. No difference in the proportion of subpatent infections was observed between seasons (dry: $73.5 \%$ (111/151), wet: $71.5 \%$ (218/305), $P=$ 0.648). $52.9 \%$ (63/119) of all infections with gametocytes detected by RT-qPCR were subpatent across all surveys, with equal proportions in the dry $(52.9 \%(27 / 51))$ and wet season $(52.9 \%(36 / 68))$. Mean $p f s 25$ densities were 3 -fold lower in subpatent infections compared to patent infections ( 1.26 vs. 3.64 transcripts $/ \mu \mathrm{L}, P=0.003$ ).

\section{Discussion}

We observed a contrasting pattern of gametocyte carriage between the dry and the wet season in blood samples collected from 2859 afebrile individuals residing in a malaria endemic area of western Kenya. In the wet season, when most transmission is expected to occur, fewer infections harbored gametocytes. Among gametocytepositive infections, however, gametocyte densities were higher, as was the proportion of infections harboring gametocytes at densities that could likely infect mosquitos. The higher gametocyte densities in the wet season are particularly noteworthy as parasite densities did not differ between seasons. Thus, the proportion of gametocytes among total blood stage parasites was higher in the wet season compared to the dry season. Our results imply that parasites increase their investment in gametocytes in the high transmission period to be synchronized with increased vector abundance in the rainy season.

However, the adjustment was not uniform across all infections. Less than a quarter of infections carried detectable gametocytes in the wet season. This is line with previous studies, where a majority of infections did not carry gametocytes detected by RT-qPCR [44, 45]. In some cases gametocytes might be present below the limit of detection even by RT-qPCR [46]. Yet, even among medium-to-high density infections (above 100 parasites $/ \mu \mathrm{L}$ ), more than half did not carry gametocytes. Given the high sensitivity of our RT-qPCR, limited detectability cannot explain this result.

Presence of $p f s 25$ transcripts detected by RT-qPCR does not necessarily imply infectivity. Molecular methods detect transcripts at densities at below the limit for successful mosquito infections [47], and the proportion of infections with detectable transcripts depends on the limit of detection of the molecular assay $[46,48]$. Gametocyte density, and the proportion of infections with gametocytes at a density that could infect mosquitos appear to be more informative measures [23, 25]. At low gametocyte densities, mosquito infectivity increases with increase in gametocyte density. At high densities of several hundred gametocytes per uL blood, infectivity reaches saturation [25], yet very few infections in the present study were in this range.

While our quantification of $p f s 25$ transcripts is a good marker of infectivity at time of sample collection [23, 25, 49 ], it is only an indirect measure of commitment to transmission. Asexual parasite densities are expected to peak early in the infection, when mature gametocytes are not yet circulating. Likely, some of the high-density infections observed in our study were recently acquired and carried sequestered gametocytes that appeared in the blood a few days after sample collection. Among infections with above average proportions of gametocytes, asexual densities might have been higher two weeks prior when gametocyte development was initiated. Alternatively, the pattern might reflect true differences in gametocyte conversion. Few studies have measured the conversion rate directly on field isolates, and those who did found pronounced variation among $P$. falciparum isolates [32-34]. The factors underlying these differences remain poorly understood.

Our findings of higher gametocyte densities in the wet season are in line with xenodiagnostic surveys conducted from asymptomatic residents of Burkina Faso and Kilifi, Kenya. Gametocyte densities determined by molecular assays targeting $p f s 25$ transcripts and infectivity were substantially higher in the wet compared to the dry season [23, 24]. Similarly, the present study corroborates previous work on asymptomatic individuals in eastern Sudan [50]. These adjustments to seasonality have important implications for programs that aim to detect asymptomatic infections through population screening. In all surveys for the present study, $67-80 \%$ of infections were calculated to be subpatent $(<100$ parasites/uL). In both sites and seasons, approximately half of all individuals that had gametocyte detected by RT-qPCR carried infections at densities below the limit of detection of microscopy or rapid diagnostic test. They thus would escape screening of asymptomatic individuals using field-deployable diagnostics. Gametocyte densities were 3-fold lower in subpatent individuals, yet among the 30 infections with moderate to high gametocyte densities, 11 were subpatent. Among them, 8 were sampled in the wet season. Thus, population screening would miss a much larger proportion of infections likely infective in the wet season compared to the dry season. 
As opposed to Chulaimbo where parasite prevalence doubled in the wet season, in Homa Bay the prevalence did not change. The variations in seasonal parasite prevalence pattern between Chulaimbo and Homa Bay may be due to differences in species composition of local vector populations [51]. In Chulaimbo, An. Arabiensis forms the predominant mosquito vector species followed by Anopheles gambiae s.s [5]., whereas in Homa Bay An. funestus is the predominant mosquito vector species [37]. An. funestus prefers permanent bodies of water like irrigated rice fields that last beyond the wet seasons, while $A n$. arabiensis prefers temporary holes and pools that dry out once the rainy season ends [52-55].

In conclusion, we have observed changes in the investment in transmission across seasons in asymptomatic $P$. falciparum infections in two sites. The increased infectivity in the wet season has important implications for control interventions. Given that it is not paralleled by increased parasite densities, screening using RDT or light microscopy in the wet season would miss an even larger proportion of the infectious reservoir than in the dry season. A small number of individuals, mostly school children, carried high gametocyte densities and likely contributed disproportionally to transmission. Targeted treatment of school children at the beginning of the wet season with gametocidal drugs such as low-dose primaquine in addition to blood-stage treatment might reduce transmission substantially [56]. Given the limited sensitivity of microscopy or RDT, this treatment should not be based on field-deployable diagnosis. Further research will be required to understand the stimuli that cause parasites to increase gametocyte density in the wet season, such as the frequency of uninfected mosquito bites [57], or they might sense physiological factors of the human body that change in response to seasonality. Surveillance systems assessing the impact of control on malaria asymptomatic reservoirs need to consider seasonal changes of gametocytemia that might differ from changes in parasitemia.

\section{Supplementary Information}

The online version contains supplementary material available at https://doi. org/10.1186/s12879-020-05761-6.

Additional file 1. $q P C R, R T-q P C R$, and ddPCR conditions

Additional file 2. Database.

\section{Abbreviations}

IRS: indoor residual spraying; RT-qPCR: reverse transcriptase - quantitative polymerase chain reaction; ddPCR: droplet digital PCR; varATS: var gene acidic terminal sequence; RNA: ribonucleic acid; DNA: deoxyribonucleic acid; EDTA: ethylenediaminetetraacetic acid

\section{Acknowledgements}

We are grateful to all study participants and thank the field teams of the sub-Saharan Africa ICEMR for support with sample collection. This work is published with the permission of the Director of KEMRI.

\section{Authors' contributions}

$\mathrm{COO}$, Conceptualization, Formal analysis, Investigation, Methodology, Data curation, Writing-original draft and revising the article; SO, Supervision, Writing-review and editing; $\mathrm{HA}$, Project administration, Writing-review and editing; BNO, Supervision, Writing-review and editing; M-CL, AKG, Project administration, Writing-review and editing; AED, Conceptualization, Project administration, Writing-review and editing; JWK, GY, Conceptualization, Supervision, Funding acquisition, Project administration, Writing-review and editing; CK, Conceptualization, Investigation, Methodology, Supervision, Formal analysis, Data curation, Writing-review and editing, Project administration, Resources. All authors read and approved the final manuscript.

Funding

This work was supported by the National Institutes of Health [U19 Al129326, D43 TW001505, R01 Al050243, R01 Al130131]. The funders had no role in study design, data collection and analysis, decision to publish or preparation of the manuscript.

\section{Availability of data and materials}

All data generated or analyzed during this study are included in this published article and its supplementary information files.

\section{Ethics approval and consent to participate}

Ethical approval to conduct the study was obtained from Maseno University Ethics Review Committee (MUERC protocol number 00456), the University of California, Irvine Institutional Review Board (HS\# 2017-3512), and the University of Notre Dame. All study participants and guardians of minors gave informed written consent prior to obtaining clinical and demographic information and drawing a blood sample.

\section{Consent for publication}

Not applicable.

\section{Competing interests}

The authors declare that they have no competing interests.

\section{Author details}

'Department of Biochemistry and Molecular Biology, Egerton University, P. O Box 536, Nakuru 20115, Kenya. ${ }^{2}$ Kenya Medical Research Institute/Centre for Global Health Research, P. O Box 1578, Kisumu 40100, Kenya. ${ }^{3}$ School of Public Health, Maseno University, P. O Box 3275, Maseno 40100, Kenya.

${ }^{4}$ International Center of Excellence for Malaria Research, P. O Box 199, Homa Bay 40300, Kenya. ${ }^{5}$ Laboratory of Malaria Immunology and Vaccinology, National Institute of Allergy and Infectious Diseases, National Institute Health, Bethesda, MD 20892, USA. ${ }^{6}$ Program in Public Health, College of Health Sciences, University of California, Irvine, CA 92697, USA. ${ }^{7}$ Case Western Reserve University, Center for Global Health and Diseases, LC 4983, Cleveland, $\mathrm{OH} 44106$, USA. ${ }^{8}$ Eck Institute for Global Health and Department of Biological Sciences, University of Notre Dame, Notre Dame, IN 46556-0369, USA.

Received: 26 October 2020 Accepted: 30 December 2020 Published online: 09 January 2021

\section{References}

1. McCann RS, Cohee LM, Goupeyou-Youmsi J, Laufer MK. Maximizing impact: can interventions to prevent clinical malaria reduce parasite Transmission? Trends Parasitol 2020;0. doi:https://doi.org/10.1016/.jpt.2020.07.013.

2. Stuck L, Fakih BS, Al-mafazy AH, Hofmann NE, Holzschuh A, Grossenbacher $B$, et al. Malaria infection prevalence and sensitivity of reactive case detection in Zanzibar. Int J Infect Dis. 2020;97:337-46.

3. Deng C, Huang B, Wang Q, Wu W, Zheng S, Zhang H, et al. Large-scale Artemisinin-Piperaquine mass drug administration with or without Primaquine dramatically reduces malaria in a highly endemic region of Africa. Clin Infect Dis. 2018;67:1670-6. 
4. Hsiang MS, Ntuku H, Roberts KW, Dufour M-SK, Whittemore B, Tambo M, et al. Effectiveness of reactive focal mass drug administration and reactive focal vector control to reduce malaria transmission in the low malariaendemic setting of Namibia: a cluster-randomised controlled, open-label, two-by-two factorial design trial. Lancet. 2020;395:1361-73.

5. Machani MG, Ochomo E, Amimo F, et al. Resting behaviour of malaria vectors in highland and lowland sites of western Kenya: implication on malaria vector control measures. PLoS One. 2020;15(2):e0224718.

6. Huestis DL, Lehmann T. Ecophysiology of Anopheles gambiae s.l.: persistence in the Sahel. Infect Genet Evol. 2014;28:648-61.

7. Jawara M, Pinder M, Drakeley CJ, et al. Dry season ecology of Anopheles gambiae complex mosquitoes in the Gambia. Malar J. 2008;7:156.

8. Ouédraogo AL, de Vlas SJ, Nébié I, et al. Seasonal patterns of Plasmodium falciparum gametocyte prevalence and density in a rural population of Burkina Faso. Acta Trop. 2008;105(1):28-34

9. Hamad AA, Nugud AEHD, Arnot DE, et al. A marked seasonality of malaria transmission in two rural sites in eastern Sudan. Acta Trop. 2002:83(1):71-82.

10. Sinden RE. Sexual Development of Malarial Parasites. In: Baker JR, Muller R, eds. Advances in Parasitology. Academic Press, 1983: 153-216.

11. Reece SE, Drew DR, Gardner A. Sex ratio adjustment and kin discrimination in malaria parasites. Nature. 2008;453(7195):609-14.

12. Paul REL, Coulson TN, Raibaud A, Brey PT. Sex determination in malaria parasites. Science. 2000;287(5450):128-31.

13. Bruce MC, Alano P, Duthie S, Carter R. Commitment of the malaria parasite Plasmodium falciparum to sexual and asexual development. Parasitology. 1990;100(Pt 2):191-200.

14. Hawking F, Wilson ME, Gammage K. Evidence for cyclic development and short-lived maturity in the gametocytes of Plasmodium falciparum. Trans $\mathrm{R}$ Soc Trop Med Hyg. 1971;65(5):549-59.

15. Farid R, Dixon MW, Tilley L, McCarthy JS. Initiation of gametocytogenesis at very low parasite density in Plasmodium falciparum infection. J Infect Dis. 2017;215(7):1167-74

16. Farfour E, Charlotte F, Settegrana C, Miyara M, Buffet P. The extravascular compartment of the bone marrow: a niche for Plasmodium falciparum gametocyte maturation? Malar J. 2012;11(1):285.

17. Eichner M, Diebner HH, Molineaux L, Collins WE, Jeffery GM, Dietz K Genesis, sequestration and survival of Plasmodium falciparum gametocytes: parameter estimates from fitting a model to malariatherapy data. Trans $\mathrm{R}$ Soc Trop Med Hyg. 2001;95(5):497-501.

18. Lensen A, Bril A, van de Vegte M, van Gemert GJ, Eling W, Sauerwein R. Plasmodium falciparum: infectivity of cultured, synchronized gametocytes to mosquitoes. Exp Parasitol. 1999;91(1):101-3.

19. Smalley ME, Sinden RE. Plasmodium falciparum gametocytes: their longevity and infectivity. Parasitology. 1977;74(1):1-8.

20. Rodriguez-Barraquer I, Arinaitwe E, Jagannathan P, et al. Quantification of anti-parasite and anti-disease immunity to malaria as a function of age and exposure. eLife. 2018;25:7.

21. Moormann AM, Sumba PO, Chelimo K, et al. Humoral and cellular immunity to Plasmodium falciparum Merozoite surface protein 1 and protection from infection with blood-stage parasites. J Infect Dis. 2013;208(1):149-58.

22. Nassir E, Abdel-Muhsin A-MA, Suliaman S, et al. Impact of genetic complexity on longevity and gametocytogenesis of Plasmodium falciparum during the dry and transmission-free season of eastern Sudan. Int J Parasitol. 2005;35(1):49-55.

23. Gonçalves BP, Kapulu MC, Sawa P, et al. Examining the human infectious reservoir for Plasmodium falciparum malaria in areas of differing transmission intensity. Nat Commun. 2017;8(1):1-11.

24. Ouédraogo AL, Gonçalves BP, Gnémé $A$, et al. Dynamics of the human infectious reservoir for malaria determined by mosquito feeding assays and ultrasensitive malaria diagnosis in Burkina Faso. J Infect Dis. 2016;213(1):90-9.

25. Churcher TS, Bousema T, Walker $M$, et al. Predicting mosquito infection from Plasmodium falciparum gametocyte density and estimating the reservoir of infection. eLife. 2013;2:e00626.

26. Transmission T malERA RCP on $C$ the $R$ and M. malERA: An updated research agenda for characterising the reservoir and measuring transmission in malaria elimination and eradication. PLOS Med 2017; 14(11):e1002452.

27. Gouagna LC, Ferguson HM, Okech BA, et al. Plasmodium falciparum malaria disease manifestations in humans and transmission to Anopheles gambiae: a field study in Western Kenya. Parasitology. 2004;128(3):235-43.

28. Bousema T, Okell L, Shekalaghe $\mathrm{S}$, et al. Revisiting the circulation time of Plasmodium falciparum gametocytes: molecular detection methods to estimate the duration of gametocyte carriage and the effect of gametocytocidal drugs. Malar J. 2010;9(1):136

29. Greischar MA, Mideo N, Read AF, Bjørnstad ON. Quantifying Transmission Investment in Malaria Parasites. PLoS Comput Biol. 2016;12:e1004718.

30. Mitri C, Thiery I, Bourgouin C, Paul REL. Density-dependent impact of the human malaria parasite Plasmodium falciparum gametocyte sex ratio on mosquito infection rates. Proc R Soc B Biol Sci. 2009;276(1673):3721-6.

31. Buckling A, Ranford-Cartwright LC, Miles A, Read AF. Chloroquine increases Plasmodium falciparum gametocytogenesis in vitro. Parasitology. 1999; 118(Pt 4):339-46.

32. Usui M, Prajapati SK, Ayanful-Torgby R, et al. Plasmodium falciparum sexual differentiation in malaria patients is associated with host factors and GDV1dependent genes. Nat Commun. 2019;10(1):2140.

33. Poran A, Nötzel C, Aly $O$, et al. Single-cell RNA sequencing reveals a signature of sexual commitment in malaria parasites. Nature. 2017; 551(7678):95-9.

34. Smalley ME, Brown J, Bassett NM. The rate of production of Plasmodium falciparum gametocytes during natural infections. Trans R Soc Trop Med Hyg. 1981;75(2):318-9.

35. Desai M, Buff AM, Khagayi $S$, et al. Age-specific malaria mortality rates in the KEMRI/CDC health and demographic surveillance system in Western Kenya, 2003-2010. PLoS One. 2014;9(9):e106197.

36. Idris ZM, Chan CW, Kongere J, et al. High and heterogeneous prevalence of asymptomatic and sub-microscopic malaria infections on islands in Lake Victoria, Kenya. Sci Rep. 2016;6.

37. McCann RS, Ochomo E, Bayoh MN, et al. Reemergence of Anopheles funestus as a vector of Plasmodium falciparum in Western Kenya after longterm implementation of insecticide-treated bed nets. Am J Trop Med Hyg. 2014;90(4):597-604.

38. Kenya 2018 spray report. Available at: https://www.google.com. Accessed 16 June 2020.

39. Koepfli C, Robinson LJ, Rarau P, et al. Blood-Stage Parasitaemia and Age Determine Plasmodium falciparum and P. vivax Gametocytaemia in Papua New Guinea. PLOS ONE 2015; 10(5):e0126747.

40. Wampfler R, Mwingira F, Javati $S$, et al. Strategies for detection of Plasmodium species gametocytes. PLoS One. 2013;8(9):e76316.

41. Hofmann N, Mwingira F, Shekalaghe S, Robinson L, Mueller I, Felger I. Ultrasensitive detection of Plasmodium falciparum by amplification of multi-copy Subtelomeric targets. PLoS Med. 2015;12(3):e1001788.

42. Koepfli C, Nguitragool W, Hofmann NE, et al. Sensitive and accurate quantification of human malaria parasites using droplet digital PCR (ddPCR). Sci Rep. 2016;6(1):39183.

43. Meerstein-Kessel L, Andolina C, Carrio E, et al. A multiplex assay for the sensitive detection and quantification of male and female Plasmodium falciparum gametocytes. Malar J. 2018;17(1):441.

44. Koepfli C, Ome-Kaius M, Jally S, Malau E, Maripal S, Ginny J, et al. Sustained malaria control over an 8-year period in Papua New Guinea: the challenge of low-density asymptomatic Plasmodium infections. J Infect Dis. 2017;216:1434-43.

45. Tadesse FG, van den Hoogen L, Lanke K, Schildkraut J, Tetteh K, Aseffa A, et al. The shape of the iceberg: quantification of submicroscopic Plasmodium falciparum and Plasmodium vivax parasitaemia and gametocytaemia in five low endemic settings in Ethiopia. Malar J. 2017;16:99.

46. Koepfli C, Yan G. Plasmodium gametocytes in field studies: do we measure commitment to Transmission or detectability? Trends Parasitol. 2018;34(5):378-87.

47. Tadesse FG, Meerstein-Kessel L, Gonçalves BP, Drakeley C, RanfordCartwright L, Bousema T. Gametocyte sex ratio: the key to understanding Plasmodium falciparum Transmission? Trends Parasitol. 2019;35:226-38.

48. Hofmann NE, Gruenberg M, Nate E, Ura A, Rodriguez-Rodriguez D, Salib M, et al. Assessment of ultra-sensitive malaria diagnosis versus standard molecular diagnostics for malaria elimination: an in-depth molecular community cross-sectional study. Lancet Infect Dis. 2018;18:1108-16.

49. Bradley J, Stone W, Da DF, et al. Predicting the likelihood and intensity of mosquito infection from sex specific Plasmodium falciparum gametocyte density. Krzych U, editor. eLife 2018; 7:e34463.

50. Gadalla AAH, Schneider P, Churcher TS, et al. Associations between Season and Gametocyte Dynamics in Chronic Plasmodium falciparum Infections. PLoS One 2016; 11(11).

51. Ayanful-Torgby R, Quashie NB, Boampong JN, Williamson KC, Amoah LE. Seasonal variations in Plasmodium falciparum parasite prevalence assessed by varying diagnostic tests in asymptomatic children in southern Ghana. PLoS One. 2018;13(6):e0199172. 
52. Kweka EJ, Zhou G, Munga S, et al. Anopheline Larval Habitats Seasonality and Species Distribution: A Prerequisite for Effective Targeted Larval Habitats Control Programmes. PLoS One 2012; 7(12).

53. Mala AO, Irungu LW. Factors influencing differential larval habitat productivity of Anopheles gambiae complex mosquitoes in a western Kenyan village. J Vector Borne Dis. 2011:48(1):52-7.

54. Ndenga BA, Simbauni JA, Mbugi JP, Githeko AK, Fillinger U. Productivity of malaria vectors from different habitat types in the Western Kenya highlands. PLoS One. 2011;6(4):e19473.

55. Fillinger $\mathrm{U}$, Sonye G, Killeen GF, Knols BGJ, Becker $\mathrm{N}$. The practical importance of permanent and semipermanent habitats for controlling aquatic stages of Anopheles gambiae sensu lato mosquitoes: operational observations from a rural town in western Kenya. Trop Med Int Health TM IH. 2004;9(12):1274-89.

56. Stepniewska K, Humphreys GS, Gonçalves BP, Craig E, Gosling R, Guerin PJ, et al. Efficacy of single dose primaquine with artemisinin combination therapy on $P$. falciparum gametocytes and transmission: A WWARN individual patient meta-analysis. J Infect Dis. 2020.

57. Paul RE, Diallo M, Brey PT. Mosquitoes and transmission of malaria parasites - not just vectors. Malar J. 2004;3(1):39.

\section{Publisher's Note}

Springer Nature remains neutral with regard to jurisdictional claims in published maps and institutional affiliations.

Ready to submit your research? Choose BMC and benefit from:

- fast, convenient online submission

- thorough peer review by experienced researchers in your field

- rapid publication on acceptance

- support for research data, including large and complex data types

- gold Open Access which fosters wider collaboration and increased citations

- maximum visibility for your research: over $100 \mathrm{M}$ website views per year

At BMC, research is always in progress.

Learn more biomedcentral.com/submissions 